\title{
Pharmacological Preconditioning with Diazoxide in the Experimental Hypothermic Circulatory Arrest Model
}

\author{
Henri Haapanen, MD,${ }^{1,2}$ Oiva Arvola, MD,${ }^{1}$ Johanna Herajärvi, MD,${ }^{1}$ Tuomas Anttila, MB,${ }^{1}$ \\ Hannu Tuominen, MD, PhD, ${ }^{3}$ Ulla Puistola, $\mathrm{MD}, \mathrm{PhD},{ }^{4}$ Peeter Karihtala, MD, PhD, ${ }^{5}$ \\ Kai Kiviluoma, MD, $\mathrm{PhD},{ }^{1}$ Tatu Juvonen, $\mathrm{MD}, \mathrm{PhD},{ }^{1,6}$ Vesa Anttila, $\mathrm{MD}, \mathrm{PhD}^{1,7}$
}

${ }^{1}$ Research Unit of Surgery, Anesthesia and Intensive Care, University of Oulu and Medical Research Center, Oulu; ${ }^{2}$ Department of Cardiothoracic Surgery, Great Ormond Street Hospital for Children, London, United Kingdom; Departments of ${ }^{3}$ Pathology, ${ }^{4}$ Obstetrics and Gynecology, and ${ }^{5}$ Oncology and Radiotherapy, Medical Research Center, Oulu University Hospital and University of Oulu; ${ }^{6}$ Department of Cardiac Surgery, Heart and Lung Center HUCH, Helsinki; ${ }^{7}$ Heart Center, Turku University Hospital, University of Turku, Turku, Finland

\section{ABSTRACT}

Background: Hypothermic circulatory arrest includes a remarkable risk for neurological injury. Diazoxide, a mitochondrial adenosine triphosphate-dependent potassium ion (K+ATP) channel opener, is known to have cardioprotective effects. We assessed its efficacy in preventing ischemic injury in a clinically relevant animal model.

Methods: Eighteen piglets were randomized into a diazoxide group $(n=9)$ and a control group $(n=9)$. Animals underwent 60 minutes of hypothermic circulatory arrest at $18^{\circ} \mathrm{C}$. Diazoxide $(5 \mathrm{mg} / \mathrm{kg}+10 \mathrm{~mL} \mathrm{NaOH}+40 \mathrm{~mL} \mathrm{NaCl})$ was infused during the cooling phase. Metabolic and hemodynamic data were collected throughout the experiment. After 24-hour follow-up, whole brain, heart, and kidney biopsy specimens were collected for analysis.

Results: Cerebellar Cytochrome-C and caspase- 3 activation was higher in the control group $(P=.02$ and $P=.016$, respectively). Antioxidant activity tended to be higher in the diazoxide group $(P=.099)$. Throughout the experiment, the oxygen consumption ratio was higher in the control animals $\left(P_{g}=.04\right)$, as were the lactate levels $\left(P_{\mathrm{g}}=.02\right)$. Cardiac function tended to be better in diazoxide-treated animals.

Conclusion: Diazoxide might confer neuroprotective effect as implied by the immunohistochemical analysis of the brain. Additionally, the circulatory effects of diazoxide were beneficial, supporting its neuroprotective effect.

Received November 14, 2016; received in revised form February 27, 2017; accepted February 28, 2017.

This work was supported by the Finnish Foundation for Cardiovascular Research, the Sigrid fuselius Foundation and the University of Oulu Scholarship Foundation.

Correspondence: Dr. Henri Haapanen, Great Ormond Street Hospital for Children, Department of Cardiothoracic Surgery, Great Ormond Street London, WC1N 37H, United Kingdom, +358-45-135-1856 (e-mail: henri. baapanen00@gmail.com).

\section{INTRODUCTION}

Cardiopulmonary bypass (CPB) with intervals of hypothermic circulatory arrest (HCA) enables a bloodless operating field, but there is, however, an increased risk for neurological impairment when the circulation is halted. The severity of the neurological injury correlates in particular with emergency situations and longer circulatory arrest times [Svensson 1993; Gega 2007]. Thus, there is a need for a neuroprotective tool in the field of complex cardiac and aortic surgery.

Diazoxide was found to react with adenosine triphosphate (ATP)-dependent potassium ion $\left(\mathrm{K}_{\text {ATP }}^{+}\right)$channels [Trube 1986; Standen 1989], which consequently resulted in the finding that diazoxide has a powerful cardioprotective effect [Garlid 1997; Nakai 1994]. Most interestingly, the evidence from experimental studies is also advocating the neuroprotective effect of diazoxide [Roseborough 2006; Wang 2011].

A similar $\mathrm{K}_{\text {ATP }}^{+}$channel was purified from the inner mitochondrial membrane ( $\mathrm{mK}^{+}{ }_{\text {ATP }}$ channel) [Paucek 1992], leading to the subsequent finding that diazoxide directly increases $\mathrm{K}^{+}$ fluxes in isolated mitochondria [Liu 2001]. This might prevent the self-destruction signaling pathway of the cell [Korge 2002]. The target of diazoxide is of the most interest in the context of neuroprotection, as the brain mitochondria apparently contain seven times more mK+ATP channels compared to liver and heart cells [Bajgar 2001].

The abundant production of reactive oxygen species (ROS) due to ischemia reacts with DNA, leaving a specific footprint: 8-hydroxydeoxyguanosine (8-OHdG), which can be reliably measured with a specific antibody. 8-Oxoguanine glycosylase (OGG1) removes 8-OHdG from the damaged DNA that is then secreted into the bloodstream [Valavanidis 2009; Abedin 2013]. Nuclear erythroid-related factor-2 (Nrf-2) is, in turn, a sensor for cellular redox status and is activated and translocated to the nucleus when exposed to oxidative stress. In the nucleus, it binds to the antioxidant response element, causing the transcription of multiple cytoprotective proteins and is thus related to cell protection during ischemia [Mitsuishi 2012]. Additionally, deglycase (DJ-1)/Parkinson disease protein 7 is a multifunctional redox-regulating protein associated 
with the mitigation of oxidative stress injury [Mitsumoto 2001]. Lastly, ischemic injury to the mitochondria is observed in the form of excessive cytochrome $\mathrm{C}$ release, which activates the cell death cascades, including caspase-3 activation [Zhan 2001].

In this study, we postulated that diazoxide might protect against ischemic insult and have beneficial circulatory effects. The primary end point was the immunohistochemical analysis of the brain while the secondary objective was to analyze the global effect of diazoxide and its relation to cerebral injury. The hypothesis was tested in a porcine model with 60-minute $\mathrm{HCA}$ at $18^{\circ} \mathrm{C}$ as the model allowed us to mimic clinical procedures and explore both intraoperative parameters and brain histology after ischemia.

\section{MATERIALS AND METHODS}

\section{Experimental Setting}

Eighteen female juvenile pigs from a native stock (range, 19.4-22.6 kg) were randomized into two groups using sealed envelopes: an intervention group $(\mathrm{n}=9)$ and a control group $(\mathrm{n}=9)$. An intravenous infusion of diazoxide $(5 \mathrm{mg} / \mathrm{kg}+10 \mathrm{~mL}$ $\mathrm{NaOH}+40 \mathrm{~mL} \mathrm{NaCl}$ ) was administered to the intervention group before the 60 minutes of HCA. The control group was treated in the same manner as the intervention group, and the infused solution was identical but for the absence of diazoxide.

\section{Preoperative Management}

All animals received humane care by the Principles of Laboratory Animal Care formulated by the National Society for Medical Research and with the Guide for the Care and Use of Laboratory Animals (http://www.nap.edu/catalog/5140. html). The study was approved by the Research Animal Care and Use Committee of the University of Oulu.

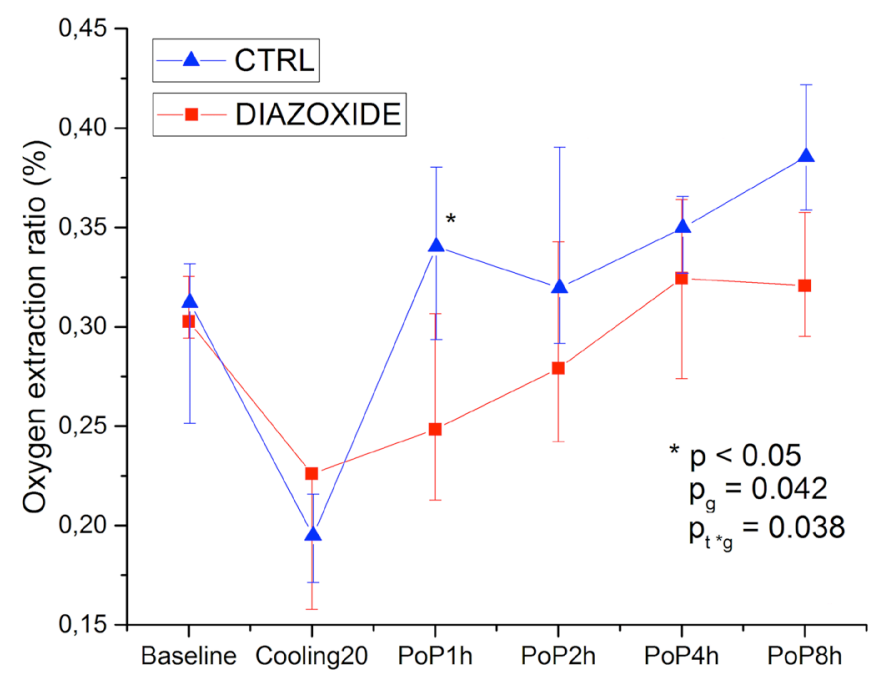

Figure 1. Oxygen consumption ratio. The oxygen consumption was significantly higher after the hypothermic circulatory arrest, whereas the diazoxide group did not reach the baseline level until 4 hours postoperatively. PoP indicates postoperatively.

\section{Anesthesia Protocol and Surgical Management}

Anesthesia induction and donor blood collection were carried out similarly as in our previous study [Jensen 2011]. Animals underwent 60 minutes of $\mathrm{HCA}$ at $18^{\circ} \mathrm{C}$. Left groin procedures for hemodynamic monitoring and CPB using $\mathrm{pH}$-stat strategy were performed as previously described in our study [Jensen 2011].

\section{Diazoxide Infusion}

Diazoxide (D9035 Sigma, Sigma-Aldrich, St. Louis, MO, USA) was first dissolved in $8-10 \mathrm{~mL}$ sodium hydroxide $(1 \mathrm{~mol} / \mathrm{L})$ and this solution was further diluted with 40 $\mathrm{mL}$ sodium chloride. The diazoxide infusion $(5 \mathrm{mg} / \mathrm{kg})$ was started at the beginning of the cooling phase, and it ended 10 minutes before HCA. The injection was performed through the pulmonary artery catheter to ensure a systemic effect.
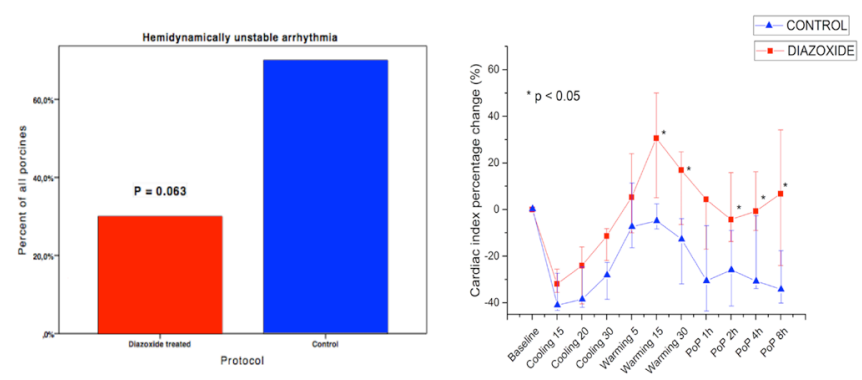

Figure 2. Cardiac output and significant arrhythmias. The left figure is showing that the hemodynamically unstable arrhythmias occurred more often in the control group. Arrhythmia was considered as unstable when the drop in mean arterial pressure was over $20 \%$ from baseline value. The heart function recovered faster in the diazoxide group as seen in cardiac index. The values are referred to baseline value and presented as percentage change. PoP indicates postoperatively.

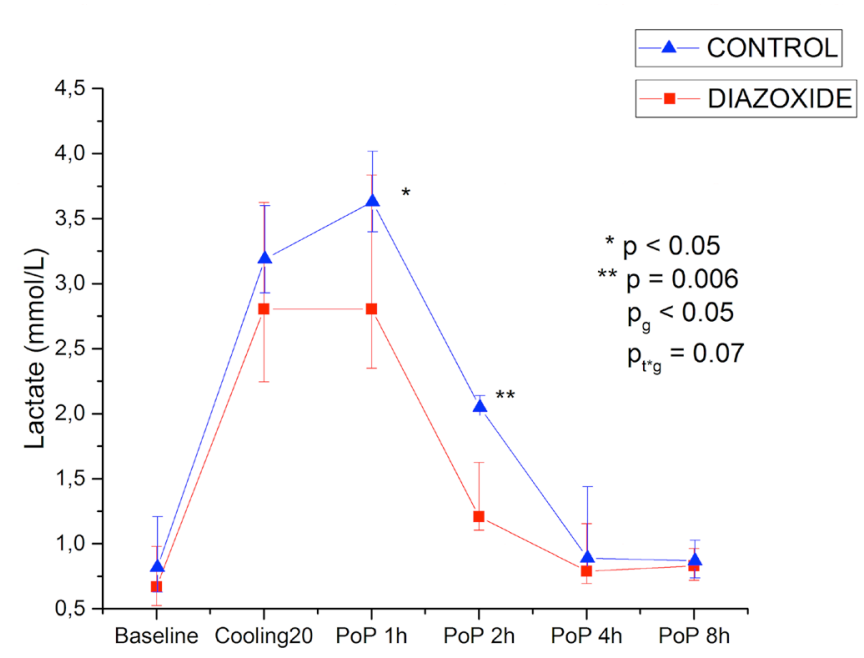

Figure 3. Venous lactate. The venous lactate levels were significantly higher in the control group after the weaning from cardiopulmonary bypass. Additionally, the decrease of lactate level was more rapid in the diazoxide group. PoP indicates postoperatively. 
The pulmonary artery catheter was rinsed with an additional $10 \mathrm{~mL}$ saline after the end of the infusion. The control group was infused with $40 \mathrm{~mL}$ saline and $10 \mathrm{~mL} \mathrm{NaOH}$ during the cooling phase of the CPB.

\section{Cranial Procedures}

A cranial window was created on the right side of the skull from the coronal suture with a $14 \mathrm{~mm} / 11 \mathrm{~mm}$ disposable cranial perforator (200-253 DGR-II, Acra Cut, Acton, MA, USA) after 8 hours from the end of HCA. The left hemisphere was biopsied and immediately immersed in $10 \%$ formalin for histopathological analysis. Hemostasis was achieved, and the skin flap was sutured.

\section{Biochemical Data}

Blood gas values and $\mathrm{pH}$, electrolyte, plasma lactate, serum ionized calcium, glucose, hematocrit, and hemoglobin levels were measured, and plasma samples were collected at baseline, at 20 minutes after the start of the cooling, 1 hour, 2 hours, 4 hours, and 8 hours after the end of HCA (i-STAT Analyzer; i-STAT Corporation, East Windsor, NJ, USA). Additionally, central temperature readings, urine amount, and the amount of fluids infused were recorded.

\section{Postoperative Management}

Mean arterial pressure was maintained at over $60 \mathrm{mmHg}$ with noradrenalin if necessary. The animals were held under general anesthesia until 24 hours from the start of HCA. At that point, each animal was electively euthanised with an injection of pentobarbital $(60 \mathrm{mg} / \mathrm{kg})$. Immediately after that, whole brain, heart, and right kidney samples were harvested and immersed in 10\% neutral formalin for two weeks.

Table 1. Metabolic Data

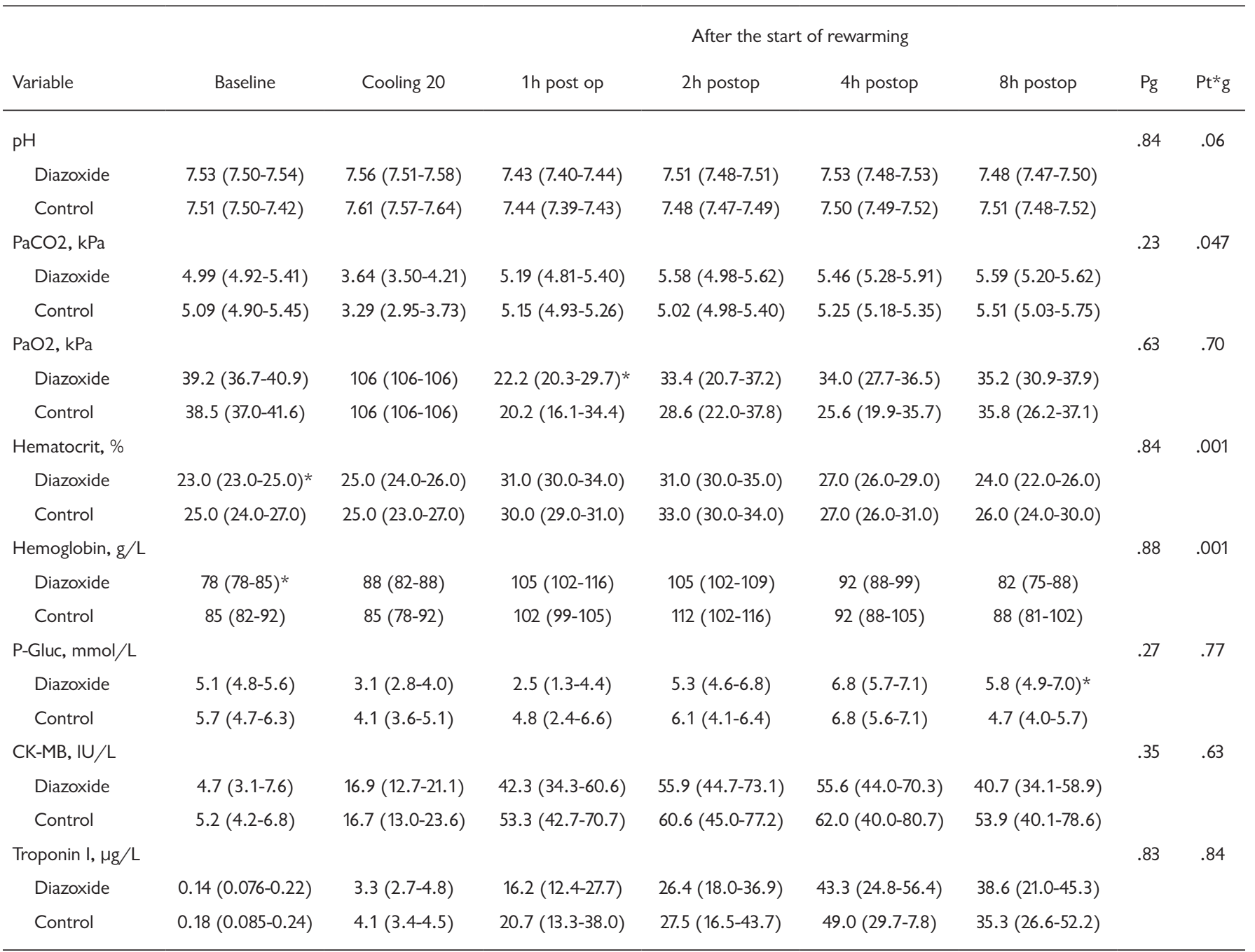

Diazoxide, $n=9$; control, $n=9$. Values are shown as medians and 25 th and 75 th percentiles. $* P<.05$ at the single time point. P-gluc, venous glucose. 


\section{Histopathological Analysis}

Hematoxylin-eosin staining was conducted as described in our previous study [Jensen 2011]. Immunohistochemical staining was used for more detailed analysis of oxidative stress and cell death. Six factors related to oxidative stress were determined: Nrf-2, OGG1, 8-OHdG, DJ-1, cytochrome C, and activated (cleaved) caspase-3.
Sections $(4.5 \mu \mathrm{m})$ cut from paraffin-embedded specimens were deparaffinized in xylene and rehydrated through graded alcohols. For antigen retrieval, the sections were pretreated with either Tris-EDTA ( $\mathrm{pH}$ 9) or with citrate buffer ( $\mathrm{pH}$ 6) in a microwave oven. After neutralizing the endogenous peroxidase activity, the sections were incubated at room temperature with diluted antibodies. Bound antibodies were

Table 2. Hemodynamic Data

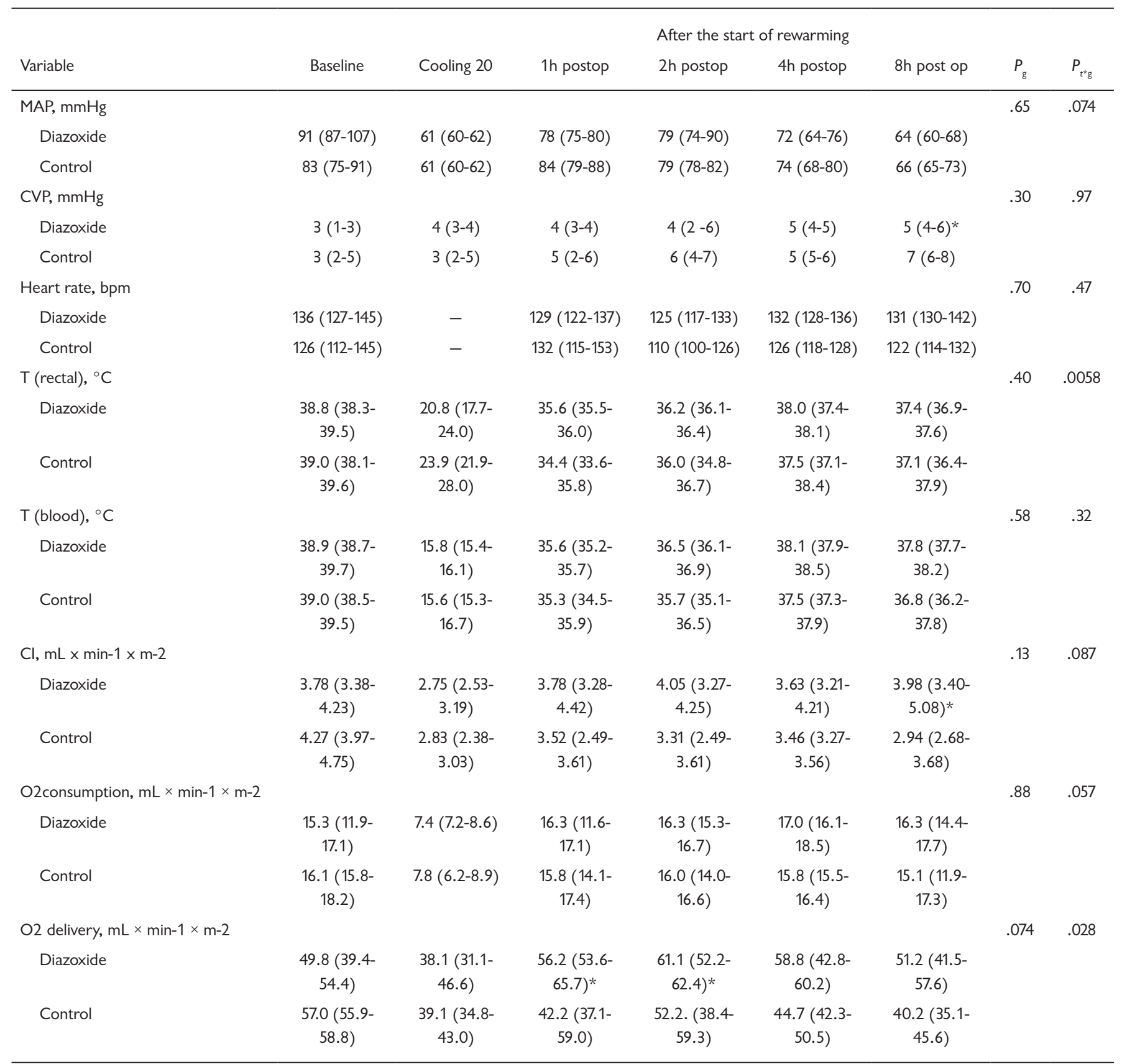

Diazoxide, $\mathrm{n}=9$; control, $\mathrm{n}=9$. Values are shown as medians and 25 th and 75 th percentiles. ${ }^{*} P<.05$ at the single time point. $\mathrm{Cl}$ indicates cardiac index; $\mathrm{CVP}$, central venous pressure; MAP, mean arterial pressure; $\mathrm{T}$, temperature. 
detected using the EnVision, EnVision FLEX (Dako, Agilent Technologies, Santa Clara, CA, USA), or Invitrogen systems (Thermo Fischer Scientific, Waltham, MA, USA). Diaminobenzidine was used as the chromogen and hematoxylin as the counterstain.

Five predetermined areas in the brains were sampled: cerebral cortex, thalamic region, cerebellum, pons, and medulla. These samples were then stained with hematoxylin-eosin and immunohistochemical stains and scored by a neuropathologist blinded to the grouping. Hematoxylin-eosin scoring was based on the presence of edema (0-3), hemorrhage $(0,2-3)$, neuron degeneration $(0,2-3)$, and the presence of infarcted tissue $(0,3)$. Immunohistochemical analysis was based on four grades as follows: $0=$ negative, $1=$ mild, $2=$ moderate, 3 = strong staining intensity. Additionally, brain biopsy, as well as kidney and heart samples, were analyzed immunohistochemically.

\section{Statistical Analysis}

Statistical analysis was performed using the SPSS (version 22.0; SPSS, Chicago, IL, USA) and SAS (version 9.2; SAS Institute, Cary, NC, USA) statistical software packages. Continuous and ordinal variables are expressed as the median and 25 th and 75 th percentiles in the tables and figures. The repeatedly measured data were analyzed using a linear mixed model with animals fitted as random effects, and the best covariance pattern was chosen according to Akaike's information criteria. Complete independence was assumed across animals (by random statement). Reported $P$ values are as follows: $\mathrm{P}$ between groups $\left(P_{\mathrm{g}}\right)$ indicates a level of difference between the groups, $P$ time $\cdot$ group $\left(P_{\mathrm{t}} \cdot \mathrm{g}\right)$ means behavior between the groups over time. Student $t$ test or the Mann-Whitney $U$ test was used as appropriate to assess the distribution of variables between the study groups. Two-tailed significance levels are reported. $P<.05$ was considered statistically significant.

\section{RESULTS}

\section{Comparability of the Study Groups}

The median weight of the animals was $20.3 \mathrm{~kg}$ in the diazoxide group and $20.7 \mathrm{~kg}$ in the control group $(P=.412)$. The diazoxide group received $76.4 \mathrm{~mL} / \mathrm{kg}$ (range, $73.2-79.8$ $\mathrm{mL} / \mathrm{kg}$ ) donor blood and the control group was given 74.5 $\mathrm{mL} / \mathrm{kg}$ (range, $69.1-78.4 \mathrm{~mL} / \mathrm{kg})$, respectively $(P=.242)$. The cumulative amount of inotropes at the end of the experiment did not differ between groups $\left(P_{g}=.750\right)$. Blood and

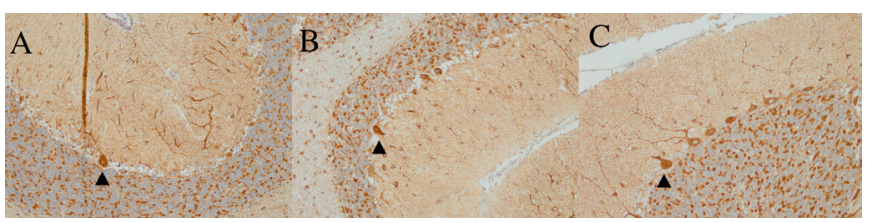

Figure 4. Immunohistochemical staining of the cerebellum revealing the activity of cytochrome $C$. The analysis was based on four grades as follows: 0 = negative, 1 = mild $(A), 2$ = moderate $(B), 3=$ strong $(C)$ staining intensity. The grading was measured from the Purkinje cells activity (arrowheads). rectal temperatures were similar throughout the experiment $\left(P_{\mathrm{g}}=.580\right.$ and $P_{\mathrm{g}}=.400$, respectively); additionally, the rectal temperature was similar during HCA $\left(P_{t . g}=0.18\right)$. Hemoglobin and hematocrit were significantly different at baseline, but the difference leveled out until the beginning of HCA (Table 1). Mortality was higher in the control group, as two animals died during the overnight surveillance $(22.2 \%$ versus $0 \%)$. The metabolic and experimental data are summarized in Tables 1 and 2.

\section{Oxygen Metabolism}

The oxygen extraction ratio (ratio of consumption and delivery) indicated that oxygen consumption was significantly higher in the control group after HCA (Figure 1). The most apparent difference was 1 hour after the start of rewarming, where a steep increase in consumption was observed. The consumption increased consistently after that in the control group, whereas in the diazoxide group, the baseline level was not reached until 4 hours after HCA.

\section{Cardiac Output and Arrbythmias}

After weaning from the CPB, the cardiac index in the diazoxide group appeared to exceed the baseline level after HCA, while it was considerably lower in the control group (Figure 2) although statistical significance was not reached $\left(P_{t^{\prime}}=.087\right)$, as presented in Table 2 . Hemodynamically unstable arrhythmias (transient ventricular tachycardia or fibrillation and atrial fibrillation with fast ventricular response) were recorded in 7 of 9 animals in the control group, whereas they were recorded in 3 of 9 animals in the diazoxide group $(P=.063)$. An arrhythmia was considered hemodynamically unstable if the decrease of MAP was over $20 \%$ of the baseline value (Figure 2).

\section{Venous Lactate}

In the diazoxide group, venous lactate levels after HCA were decreased faster compared to the control group. Moreover, the increase in the lactate concentration during and after $\mathrm{CPB}$ was not as high in the diazoxide group as in the control group (Figure 3).

\section{Histopathological Analysis}

The cytoplasmic expression of Nrf-2 tended to be more expressed in the brains of the diazoxide-treated animals (Sum score, $P=.099$ ). Cytochrome $\mathrm{C}$ and activated caspase-3 levels

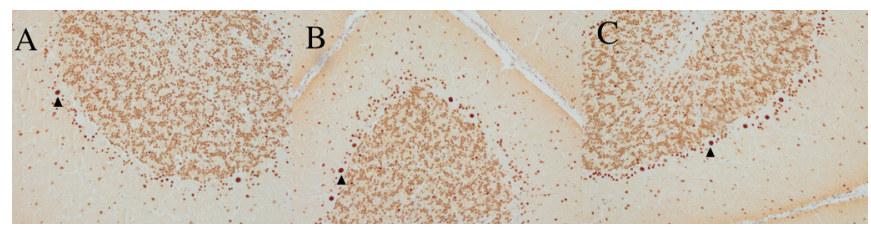

Figure 5. Immunohistochemical staining of the cerebellum revealing the activity of activated caspase 3 . The analysis was based on four grades as follows: $0=$ negative, $1=$ mild $(A), 2=$ moderate $(B), 3=$ strong (C) staining intensity. The grading was measured from the Purkinje cells activity (arrowheads). 
were higher in the cerebellum in the control group $(P=.02$ and $P=.016$, respectively). The examples of cytochrome $\mathrm{C}$ and activated caspase- 3 grading are demonstrated in Figures 4 and 5 . Levels of the oxidative stress marker $8-O H d G$ did not differ significantly between groups, and its expression was rather mild in both groups $(P=.939)$. The summarized immunohistochemical analysis is presented in Table 3 . The analysis of the heart, kidney, and cerebral biopsy were minimal and not statistically significantly different. The main findings in the hematoxylin-eosin analysis were oedema $(P=.261)$ and hemorrhage $(P=.483)$.

\section{DISCUSSION}

Hypothermia reduces the metabolic rate of cerebral tissue by approximately $50 \%$ per $6^{\circ} \mathrm{C}$ drop in tissue temperature [Kouchoukos 2013]. There has been, however, constant concern about the increased risk of neurologic deficit related to HCA [Gega 2007]. This has led to the innovation of various adjuncts to enhance the safety of HCA. In the present study, we used a clinically relevant animal model of HCA to investigate effects of diazoxide. Interestingly, it appeared diazoxide tended to enhance the antioxidant response in the brain after ischemic insult, preserved cellular metabolic capability, as well as improved cardiac function.

Initially, the Food and Drug Administration of the United States approved diazoxide for the treatment of symptomatic hypoglycemia in 1976. The intravenous form of diazoxide is also used for malignant hypertension due to its vasodilatory effect. Due to the hypotensive effect, we introduced diazoxide after the porcine was cannulated for $\mathrm{CPB}$, which enabled us to control the hemodynamics.

Several hypotheses have been proposed to explain the contribution of $\mathrm{mK}^{+}{ }_{\text {ATP }}$ channels in cellular protection. According to the isolated mitochondria studies, the leading theory of the beneficial effect of diazoxide is that the $\mathrm{mK}^{+}{ }_{\text {ATP }}$ channel opening promotes mild mitochondrial swelling, which may preserve the structure and function of the intermembrane space

Table 3. Results of Immunohistochemical Analysis of the Brain

\begin{tabular}{|c|c|c|c|c|c|c|}
\hline \multicolumn{7}{|l|}{ ৪-OHdG } \\
\hline Control & $1.0(0.0-1.0)$ & $0.0(0.0-1.0)$ & $1.0(0.5-2.0)$ & $0.0(0.0-0.5)$ & $0.0(0.0-1.0)$ & $4.0(1.0-5.0)$ \\
\hline$P$ & .900 & .243 & .619 & .414 & .243 & .939 \\
\hline Diazoxide & $1.0(1.0-2.0)$ & $1.0(1.0-2.0)$ & $2.0(2.0-3.0)$ & $2.0(2.0-3.0)$ & $1.0(1.0-1.0)$ & $9.0(7.0-10.0)$ \\
\hline Control & $2.0(1.0-2.0)$ & $2.0(1.0-2.0)$ & $3.0(2.0-3.0)$ & $2.0(2.0-2.5)$ & $2.0(1.0-2.0)$ & $10.0(9.5-10.0)$ \\
\hline$P$ & .278 & .769 & .623 & .934 & .472 & .417 \\
\hline \multicolumn{7}{|l|}{ NFR-2 } \\
\hline Diazoxide & $1.0(0.0-2.0)$ & $1.0(0.0-2.0)$ & $1.0(0.0-1.0)$ & $1.0(1.0-3.0)$ & $2.0(1.0-2.0)$ & $5.0(3.0-13.0)$ \\
\hline Diazoxide & $3.0(3.0-3.0)$ & $2.0(2.0-2.0)$ & $2.0(2.0-3.0)$ & $1.0(1.0-1.0)$ & $2.0(2.0-3.0)$ & $10.5(10.0-11.0)$ \\
\hline Control & $3.0(2.0-3.0)$ & $2.0(1.0-2.0)$ & $2.0(2.0-2.0)$ & $2.0(1.5-2.5)$ & $3.0(2.0-3.0)$ & $11.0(10.0-11.0)$ \\
\hline$P$ & .411 & .449 & .375 & .016 & .435 & .710 \\
\hline \multicolumn{7}{|l|}{ Cyt C } \\
\hline Diazoxide & $2.0(1.0-2.0)$ & $2.0(2.0-2.0)$ & $3.0(2.0-3.0)$ & $1.0(1.0-2.0)$ & $2.0(2.0-2.0)$ & $10.0(7.0-11.0)$ \\
\hline Control & $2.0(1.5-2.0)$ & $2.0(2.0-2.0)$ & $2.0(2.0-3.0)$ & $2.0(2.0-3.0)$ & $2.0(2.0-2.0)$ & $10.0(8.5-11.5)$ \\
\hline$P$ & .861 & 1.0 & .898 & .021 & .531 & .442 \\
\hline \multicolumn{7}{|l|}{ DJ-1/PARK7 } \\
\hline
\end{tabular}

Diazoxide, $n=9$; control, $n=7$. The results are presented as median values 25 th and 75 th percentiles in parenthesis. Cyt $C$ indicates cytochrome $C$. 
[Kowaltowski 2001]. Ischemia causes mitochondrial depolarization, which results in disturbance of the intermembrane space and accelerates ATP hydrolysis due to the disruption of mitochondrial creatine kinase [Kay 1997]. Thus, the mechanism of diazoxide via $\mathrm{mK}^{+}{ }_{\text {ATP }}$ channels might slow ATP hydrolysis and keep creatine kinase functional until reperfusion by preserving the intermembrane architecture. Additionally, the increase in ROS production has been associated with the use of diazoxide and $\mathrm{mK}^{+}{ }_{\text {ATP }}$ channel opening. The precise mechanism is unknown, but it has been proposed that ROS might function as a second messenger during diazoxide preconditioning as well as in ischemic preconditioning [Pain 2000].

Immunohistochemical analysis of the brain revealed the most impressive results. The statistical significance was not reached, but the tendency of more activated antioxidant pathways in the diazoxide-treated animals was apparent, although other oxidative stress markers did not show higher activity. Nrf-2 is stabilized when exposed to ROS or specific drugs, and it is translocated to the nucleus, where the transcription of various cytoprotective genes is initiated [Mitsuishi 2012]. This finding may support the theory of ROS being the second messenger, although we cannot directly conclude that because the Nrf-2 activity was found only in the cytoplasm. Interestingly, similar results have been reported from IPC studies as well [Bell 2011]. This might also explain the lack of difference in $8-\mathrm{OHdG}$, which is thought to be the most sensitive marker for DNA injury caused by ROS [Valavanidis 2009]. Moreover, cytochrome $\mathrm{C}$ release and subsequently cleaved caspase- 3 activation were higher in the cerebellum in the control group. Obviously, this indicates diazoxide might have the ability to preserve mitochondrial viability, but the difference was significant only in one brain area.

As a means to demonstrate the systemic effect of diazoxide, we documented improved oxygen consumption ratio and lower lactate levels in the diazoxide group might indicate better cellular function after ischemic insult. These findings are congruent with the suggested effect of $\mathrm{mK}^{+}{ }_{\text {ATP }}$ channel opening at the systemic level, as the reversible uncoupling of ATP hydrolysis will preserve energy stores upon reperfusion. Although these findings cannot be applied straightforwardly to the cerebral tissue protection, the beneficial systemic effects might lower the risk of cerebral injury.

The functional parameters of the heart were favorable in the diazoxide group. The better cardiac function might have a role in the neuroprotective findings of the diazoxide-treated animals, as the cerebral blood flow would be stabilized due to more efficient systemic circulation. In the first instance, ischemia-sensitive cerebral areas are compromised due to labile circulation, and thus the focal findings in cerebral immunohistochemistry may be partially explained by the circulatory effects of diazoxide.

The study carries several limitations. The model was acute, and therefore we do not have long-term surveillance and neurological, behavioral analysis. Additionally, the amount of infused diazoxide varies widely between studies from $1 \mathrm{mg} /$ $\mathrm{kg}$ to $10 \mathrm{mg} / \mathrm{kg}$ [Coetzee 2013]. There are no data on the optimal therapeutic window of diazoxide, and thus various doses might be more effective than the dose $(5 \mathrm{mg} / \mathrm{kg})$ we used. Although we cannot draw absolute conclusions about the beneficial effects of diazoxide, there are multiple parameters indicating its advantages. In addition to the biochemical and histological findings, there were two deaths in the control group, which were most probably related to heart failure, supporting the benefits of diazoxide.

In conclusion, the present study showed the beneficial effects of diazoxide against global ischemia as well as evidence of mitigated cerebral injury. Additionally, we were able to observe indirect indications of preserved mitochondrial function in a clinically relevant animal model.

\section{ACKNOWLEDGEMENT}

We would like to thank Pasi Ohtonen, MSc, for his expert help with statistical analysis and Seija Seljanperä, RN, for her help in caring for the animals.

\section{REFERENCES}

Abedin Z, Louis-Juste M, Stangl M, Field J. 2013. The role of base excision repair genes OGG1, APN1 and APN2 in benzo[a]pyrene-7,8-dione induced $\mathrm{p} 53$ mutagenesis. Mutat Res 750:121-8.

Bajgar R, Seetharaman S, Kowaltowski AJ, Garlid KD, Paucek P. 2001. Identification and properties of a novel intracellular (mitochondrial) ATP-sensitive potassium channel in brain. J Biol Chem 276:33369-74.

Bell KF, Fowler JH, Al-Mubarak B, Horsburgh K, Hardingham GE. 2011. Activation of Nrf2-regulated glutathione pathway genes by ischemic preconditioning. Oxid Med Cell Longev 2011:689524.

Coetzee WA. 2013. Multiplicity of effectors of the cardioprotective agent, diazoxide. Pharmacol Ther 140:167-75.

Garlid KD, Paucek P, Yarov-Yarovoy V, et al. 1997. Cardioprotective effect of diazoxide and its interaction with mitochondrial ATP-sensitive $\mathrm{K}+$ channels. Possible mechanism of cardioprotection. Circ Res 81:1072-82.

Gega A, Rizzo JA, Johnson MH, Tranquilli M, Farkas EA, Elefteriades JA. 2007. Straight deep hypothermic arrest: experience in 394 patients supports its effectiveness as a sole means of brain preservation. Ann Thorac Surg 84:759-66; discussion 766-7.

Jensen HA, Loukogeorgakis S, Yannopoulos F, et al. 2011. Remote ischemic preconditioning protects the brain against injury after hypothermic circulatory arrest. Circulation 123:714-21.

Kay L, Rossi A, Saks V. 1997. Detection of early ischemic damage by analysis of mitochondrial function in skinned fibers. Mol Cell Biochem 174:79-85.

Korge P, Honda HM, Weiss JN. 2002. Protection of cardiac mitochondria by diazoxide and protein kinase C: implications for ischemic preconditioning. Proc Natl Acad Sci U S A 99:3312-17.

Kouchoukos NT, Blackstone EH, Hanley FL, Kirklin JW. 2013. Hypothermia, circulatory arrest and cardiopulmonary bypass. In: Cardiac surgery: morphology, diagnostic criteria, natural history, techniques, results and indications. Philadelphia: Elsevier. pp. 67-133.

Kowaltowski AJ, Seetharaman S, Paucek P, Garlid KD. 2001. Bioenergetic consequences of opening the ATP-sensitive $\mathrm{K}(+)$ channel of heart mitochondria. Am J Physiol Heart Circ Physiol 280:H649-57.

Liu Y, Ren G, O’Rourke B, Marban E, Seharaseyon J. 2001. 
Pharmacological comparison of native mitochondrial K(ATP) channels with molecularly defined surface K(ATP) channels. Mol Pharmacol 59:225-30.

Mitsuishi Y, Motohashi H, Yamamoto M. 2012. The Keap1-Nrf2 system in cancers: stress response and anabolic metabolism. Front Oncol 2:200.

Mitsumoto A, Nakagawa Y. 2001. DJ-1 is an indicator for endogenous reactive oxygen species elicited by endotoxin. Free Radic Res 35:885-93.

Nakai T, Ichihara K. 1994. Effects of diazoxide on norepinephrineinduced vasocontraction and ischemic myocardium in rats. Biol Pharm Bull 17:1341-4.

Pain T, Yang XM, Critz SD, et al. 2000. Opening of mitochondrial $\mathrm{K}(\mathrm{ATP})$ channels triggers the preconditioned state by generating free radicals. Circ Res 87:460-6.

Paucek P, Mironova G, Mahdi F, Beavis AD, Woldegiorgis G, Garlid KD. 1992. Reconstitution and partial purification of the glibenclamidesensitive, ATP-dependent $\mathrm{K}+$ channel from rat liver and beef heart mitochondria. J Biol Chem 267:26062-9.

Roseborough G, Gao D, Chen L, et al. 2006. The mitochondrial K-ATP channel opener, diazoxide, prevents ischemia-reperfusion injury in the rabbit spinal cord. Am J Pathol 168:1443-51.
Standen NB, Quayle JM, Davies NW, Brayden JE, Huang Y, Nelson MT. 1989. Hyperpolarizing vasodilators activate ATP-sensitive K+ channels in arterial smooth muscle. Science 245:177-80.

Svensson LG, Crawford ES, Hess KR, et al. 1993. Deep hypothermia with circulatory arrest. Determinants of stroke and early mortality in 656 patients. J Thorac Cardiovasc Surg 106:19-28; discussion 28-31.

Trube G, Rorsman P, Ohno-Shosaku T. 1986. Opposite effects of tolbutamide and diazoxide on the ATP-dependent $\mathrm{K}+$ channel in mouse pancreatic beta-cells. Pflugers Arch 407:493-9.

Valavanidis A, Vlachogianni T, Fiotakis C. 2009. 8-hydroxy-2' -deoxyguanosine (8-OHdG): A critical biomarker of oxidative stress and carcinogenesis. J Environ Sci Health C Environ Carcinog Ecotoxicol Rev $27: 120-39$

Wang L, Zhu QL, Wang GZ, et al. 2011. The protective roles of mitochondrial ATP-sensitive potassium channels during hypoxia-ischemiareperfusion in brain. Neurosci Lett 491:63-7.

Zhan RZ, Wu C, Fujihara H, et al. 2001. Both caspase-dependent and caspase-independent pathways may be involved in hippocampal CA1 neuronal death because of loss of cytochrome c From mitochondria in a rat forebrain ischemia model. J Cereb Blood Flow Metab 21:529-40. 